\title{
Evaluation of the prognostic value of 18F-FDG- PET/CT parameters in head and neck cancer patients treated with definitive intensity modulated radiotherapy
}

Mitsue Kawamura ( $\nabla$ mitkawa@kuhp.kyoto-u.ac.jp )

Kyoto University

Michio Yoshimura

Kyoto University

Takashi Mizowaki

Kyoto University

\section{Research Article}

Keywords:

Posted Date: February 3rd, 2022

DOI: https://doi.org/10.21203/rs.3.rs-1322351/v1

License: (c) (1) This work is licensed under a Creative Commons Attribution 4.0 International License.

Read Full License 


\section{Abstract \\ Purpose}

To investigate the treatment outcome and prognostic factor of volumetric parameters measured by $18 \mathrm{~F}-$ FDG-PET/CT in squamous cell carcinoma patients of oropharynx, hypopharynx, and larynx treated with definitive intensity modulated radiotherapy.

\section{Methods}

We retrospectively reviewed 63 patients who received definitive radiation therapy for head and neck squamous cell carcinoma (HNSCC) between April 2007 and March 2012 in our hospital. Clinical factor and 18F-FDG-PET/CT parameters were evaluated using univariate and multivariate Cox regression analysis.

\section{Result}

With a median follow up of 61 months (range 4-116 month), the 5-year overall survival (OS), progressionfree survival (PFS) rate was $58.2 \%$, and $63.0 \%$, respectively. Recurrence was observed in 24 patients. Seventeen patients had locoregional recurrence and 9 patients developed distant metastasis. ROC analysis revealed the best threshold of pretreatment FDG-PET parameters was 14.8 of SUVmax, $5.4 \mathrm{ml}$ of metabolic tumor volume (MTV), and $38.5 \mathrm{ml}$ of total lesion glycolysis (TLG). On univariate analysis, MTV $(>5.4 \mathrm{ml})$, and TLG $(>38.5 \mathrm{ml})$ were predictive of poor OS $(p<0.01, p<0.01)$ and poor DFS $(p=0.01, p<0.01)$. On multivariate analysis, MTV displayed significantly worse effects on OS and DFS ( $p=0.02,<0.01$, respectively), while SUVmax was nonsignificant ( $p=0.70$ for OS, $p=0.70$ for DFS).

\section{Conclusion}

This study showed that18F-FDG-PET/CT parameters are useful as prognostic factor of definitive intensity modulated radiotherapy of HNSCC. These prognostic factors may identify the population who have benefit from high intensity treatment, ex. dose escalation planning.

\section{Introduction}

Radiation therapy is one of principal treatments for head-and neck carcinoma (HNC). Definitive chemoradiotherapy in patients with advanced HNC can preserve laryngopharyngeal function such as speaking, swallowing, and breathing. Radiotherapy of head-and-neck cancers needs to minimize radiation exposure to a large number of organs-at-risk (OARs). Intensity modulated radiotherapy (IMRT) can reduce complication and escalate dose prescription [1]. Improvements in identification of the tumor volume of head and neck tumors using imaging such as ${ }^{18} \mathrm{~F}$ labeled Fludeoxyglucose positron emission 
tomography with computed tomography (18F-FDG-PET/CT) have facilitated radiation treatment planning by improved target volume delineation and more accurate target localization, which is critical for IMRT planning. Sharp dose gradients between the high dose region targeted at the tumor and adjacent low dose normal tissue regions in IMRT improved the therapeutic ratio between tumor control and radiation related toxicity, although this is reliant on the accurate identification of the tumor extent.

18F-FDG-PET/CT is a medical imaging technique based on the study of glucidic metabolism of tumor cells [2]. During the last decade, it has emerged as an essential imaging tool in the field of oncology, not only for diagnosis but also for prognostic and therapeutic evaluation [3,4]. Concerning HNSCC disease, there have been many studies investigating the prognostic value $18 \mathrm{~F}-\mathrm{FDG}-\mathrm{PET} / \mathrm{CT}$. In fact, the maximum standardized uptake value (SUVmax), a semi-quantitative measure of tumoral uptake, is a predictor of survival, regardless of the size and stage of the tumor but without a real cut-off set, varying between 4 and 10 according to previous studies [5,6]. On the other hand, metabolic tumor volume (MTV) defined as the volume of FDG activity in a tumor assessed by automated volume of interest (VOI) delineation has been proposed as a quantitative PET index. MTV has been reported as an additional diagnostic [7] and prognostic imaging biomarker in various solid cancers [8]. A large MTV has been already suggested as a poor prognostic factor in HNSCC, and even seems a better predictor of survival than SUVmax [9].

The relative importance between SUV, MTV, and total lesion glycolysis (TLG) of the primary tumor in the risk stratification of HNSCC patients has not been determined. In our study, we analyze recurrence predictor using 18F-FDG-PET/CT parameters in head and neck squamous cell carcinoma treated with definitive IMRT and try to determine which population needs dose escalation or dose painting.

\section{Materials And Methods}

\section{Patients}

Retrospectively, we analyzed consecutive 63 patients with histologically proven oropharyngeal and hypopharyngeal squamous cell carcinoma. They underwent definitive IMRT in our institution from April 2007 to March 2011. All patients had 18F-FDG PET/CT image data from multi-institution when we planned IMRT.

The primary sites were the oropharynx in 16 patients, and hypopharynx in 37 . The median patient age was 61 years (range 40-91 years) and the 48 patients are male. The patients' characteristics are shown in Table I. The patients were staged according to the malignant tumour criteria of the Union for International Cancer Control (6th edition) [10]. The clinical stage was I, II, III, IVA, and IVB in 5, 8, 4, 33, and 3 patients, respectively. Written informed consent was obtained from all patients and the study was approved by the local ethics committee.

\section{Treatment}


Definitive radiotherapy was carried out using standard dose fractions. All patients were treated with photon beam. Simultaneous integrated boost (SIB)-IMRT was used in all cases. The prescription dose was 70Gy in 2Gy fractions for primary tumor and positive lymph node, and 63Gy in 1.8Gy fractions for high-risk clinical target volume (CTV), and 56Gy in $1.6 \mathrm{~Gy}$ fractions for low-risk CTV.

Twenty-one patients received neoadjuvant chemotherapy and 48 patients received concurrent chemotherapy, and 10 patients received neck dissections before radiotherapy.

\section{PET/CT parameters}

All patients underwent the initial PET/CT before treatment. Because patients received PET/CT at multicenter institutions, the study protocol is uncertain in detail. To revise the multicenter value, we calculate TBR in addition to SUVmax. The PET images were displayed on a workstation (MIM maestro, version 6.5.3). We used auto-contouring program by thresholds.

\section{Statistical Analysis}

Statistical analyses were performed using EZR ver. 1.31 software (Saitama Medical Center, Jichi Medical University, Saitama, Japan), which is a graphical user interface for R (the R Foundation for Statistical Computing, Vienna, Austria) [11]. We examined the association between patient outcome and PET/CT parameters using the univariate Cox proportional hazards regression analysis.

For assessment of patient outcome, we calculated overall survival (OS), disease-free survival (DFS), and loco-regional control (LRC) rates, and distance control (DC) rate. Outcomes were calculated as the time between the start of radiotherapy and the event or the last clinical follow-up.

\section{Result}

The median follow-up period was 61 months (range, 4-116 months) . In total, 24 patients had disease recurrence and 26 patients died during follow-up. The median OS and DFS were 77.2 month [95\% confidence interval (Cl): 40.4-NA] and 105.7 months [95\% Cl: 19.6-NA], respectively. The 5-year OS and PFS were $58.2 \%$ and $63.0 \%$, respectively. Patient characteristics are shown in Table 1. Details of the neoadjuvant and concurrent chemotherapy and neck dissections are also described.

\section{FDG PET/CT findings and Patient Outcome}

The thresholds of each parameter were determined according to the ROC analysis results, and optimal cutoffs of $14.8,8.7,5.4 \mathrm{ml}$ and $38.5 \mathrm{ml}$ were chosen for SUVmax, TBR, MTV, and TLG, respectively. We performed subgroup analysis using the Kaplan-Meier method and log-rank tests to evaluate the differences between groups, and MTV and TLG were found to be significant predictors of OS, DFS, LRC, and DC (MTV; $p=0.002,<0.001,0.025$, and 0.008, respectively, TLG; $p=0.013,0.005,0.029$, and 0.104, respectively) as shown in Figure 1, and Table 2. 


\section{Recurrence pattern and localization of local failure}

At the time of data cut-off (28 May 2017), PFS events were observed in 24 patients and 30 patients had dead. The local recurrence was occurred in 10 patients. As for local recurrence, MTV and TLG were significant predictors (all, $p<0.01$ ) (Figure 2). In 5 of the 10 patients whose 18F-FDG-PET were available, the recurrence tumor volume was included in initial clinical target volume, and the distance between the two points of the SUVmax of initial target volume and recurrence volume was mean $1.4 \pm S D 0.5 \mathrm{~cm}$. Representative images from patients are shown in Figure 3.

\section{Discussion}

This study showed that MTV and TLG are superior prognostic biomarkers of treatment outcome and survival for HNSCC patients undergoing definitive IMRT as compared to SUVmax or TBR. MTV will be useful to identify a subgroup of patients with a poor diagnosis who may benefit from aggressive therapy such as dose escalation aiming to improve their survival.

Schwartz et al. [12] evaluated 54 patients with HNSCC, undergoing RT including postoperative patients with or without concurrent chemotherapy, and reported that a SUV of greater than 9, the median, significantly correlated with inferior local control and disease-free survival. On univariate and multivariate analyses these data remained significant or borderline significant. Similarly, Machtay et al. [13] reported in a cohort of $60 \mathrm{HNSCC}$ patients, treated with definitive radiotherapy with or without concurrent chemotherapy, that an SUVmax $<9$, median SUVmax of the study was 7.2, was associated with improved 2 -year DFS of 72 versus $37 \%(p=0.007)$. Torizuka et al. [14] reported in 50 consecutive HNSCC patients who underwent definitive RT with or without chemotherapy, or surgery with or without postoperative RT that an SUVmax of B7 significantly predicted higher rates of 2-year local control and disease-free survival. When adjusted for age and nodal stage these findings remained significant. However, the median SUVmax for the cohort was 10.53, and they did not identify how an SUV max of 7 was selected as the optimal cut point. Limitations of comparing SUV as a radiological biomarker between studies includes the use of different SUV cutoff values which may be influenced by multiple factors including patient selection, differences in imaging technique, injected FDG dose, incubation period, protocol, scanner, and reconstruction algorithm variation [15-17].

As time passed by, MTV and TLG was defined in 2009 and 2011, respectively [18, 19]. MTV is defined as the volume of the tumor demonstrating FDG uptake. It represents a volumetric and metabolic biomarker, and estimates tumor volume based on the distribution of metabolic activity. Therefore, unlike SUVmax, which is a single-pixel representation of the maximum FDG uptake by the tumor, MTV quantifies the overall tumor burden [20]. Thus, volume-based parameters, such as MTV, were sought in hopes of identifying more accurate ways to prognosticate disease. TLG is defined as the tumor volume multiplied by SUVmean of included voxels. Because this parameter incorporates both the MTV and SUV, it represents both the degree of FDG uptake and the size of the tumor. Like MTV, TLG theoretically 
represents the total activity of the metabolically active cancer cells. It should be an ideal representation of overall tumor burden. La et al. [21] found that only tumor volume as measured on PET, not the SUV, was associated with progression free survival and overall survival in patients with head and neck cancer. These results are similar to our findings that the MTV was a more accurate predictor than the SUVmax with regard to response to treatment and the progression free survival. The significance of being able to stratify patients according to their MTV at diagnosis into two distinct outcome groups suggests that its specific value is significant, and that the MTV might be a quantitative biomarker for predicting which patients will have a worse outcome before treatment is initiated.

This study had some limitations. First, because this was a retrospective study in a single institute, information bias was inevitable, and sample size was small. The heterogeneity of the primary tumor site, the high population of advanced stage, and the nonuniform the treatment regimens. Second, the human papilloma virus (HPV) status of the oropharyngeal lesions was not determined, because at the start of this study HPV/p16 status was not examined routinely in our institution. Despite these limitations, we obtained significant results indicating that MTV and TLG was an important prognostic factor in patients with HNSCC.

Recently, in addition to these general features such as SUVmax, MTV, and TLG, textural feature analysis as part of radiomics is an emerging field in quantitative medical imaging analysis, allowing to extract higher dimensional features from grey level distribution of image that are unrecognizable by visual inspection as a further approach.

\section{Conclusion}

This study showed that MTV and TLG are superior prognostic utility of treatment outcome and survival for HNSCC patients undergoing definitive IMRT as compared to SUVmax or TBR. MTV will be useful to identify a subgroup of patients with a poor diagnosis who may benefit from aggressive therapy such as dose escalation aiming to improve their survival.

\section{Declarations}

\section{Acknowledgements}

This work was supported by Grants-in-Aid for Scientific Research (A) (25253078) from the Japan Society for the Promotion of Science.

\section{Conflict of Interest}

We have no financial relationships to disclose. 


\section{Ethical statement:}

This study was approved by the local ethical committee of Kyoto University Hospital.

Presentation: Presented at the 2017 annual meeting of the Japan Society of Clinical Oncology, Yokohama, Japan.

\section{References}

[1] Hong TS, Tome WA, Harari PM. Intensity-modulated radiation therapy in the management of head and neck cancer. Curr Opin Oncol 2005;17:231-235.

[2] Phelps ME, Huang SC, Hoffman EJ, Sokoloff L, Kuhl DE. Tomographic measurement of local cerebral glucose metabolic rate in humans with (18-F)2-fluoro-2-deoxy-D-glucose: validation of method. Ann Neurol. 1979;6:371-88.

[3] Fletcher JW, Djulbegovic B, Soares HP, Siegel BA, Lowe VJ, Lyman GH, et al. Recommendations on the use of 18F-FDG PET in oncology. J Nucl Med. 2008;49:480-508.

[4] Bourguet P, Blanc-Vincent MP, Boneu A, Bosquet L, Chauffert B, Corone C, et al. Summary of the Standards, Options and Recommendations for the use of positron emission tomography with 2[18F]fluoro-2-deoxy-D-glucose (FDP-PET scanning) in oncology (2002). Br J Cancer. 2003;89:84-91.

[5] Halfpenny W, Hain SF, Biassoni L, Maisey MN, Sherman JA, McGurk M. FDG-PET. A possible prognostic factor in head and neck cancer. Br J Cancer. 2002;86:512-6.

[6] Allal AS, Dulguerov P, Allaoua M, Haengelli CA, el El-Ghazi A, Lehmann W, et al. Standardized uptake value of 2-[18F]fluoro-2-deoxy-D-glucose in predicting outcome in head and neck carcinomas treated by radiotherapy with or without chemotherapy. J Clin Oncol. 2002;20:1398-404.

[7] Dibble EH, Alvarez AC, Truong MT, Mercier G, Cook EF, Subramaniam RM. 18F-FDG metabolic tumor volume and total lesion glycolytic activity of oral cavity and oropharyngeal squamous cell cancer: adding value to clinical staging. J Nucl Med. 2012;53:709-15.

[8] Moon SH, Hyun SH, Choi JY. Prognostic significance of volumebased PET parameters in cancer patients. Korean J Radiol. 2013;14:1-12.

[9] Abgral R, Koromnes N, Robin P, et al. Prognostic value of volumetric parameters measured by ${ }^{18} \mathrm{~F}-\mathrm{FDG}$ $\mathrm{PET} / \mathrm{CT}$ in patients with head and neck squamous cell carcinoma. Eur J Nucl Med Mol Imaging. 2014;41:659-667. 
[10] L.H. Sobin, C.Wittekind. TNM Classification of malignant tumors (UICC International Union Against Cancer) (6 $6^{\text {th }}$ edition) Wiley-Blackwell, Oxford, 2002.

[11] Kanda Y. Investigation of the freely-available easy-to-use software "EZR" (Easy R) for medical statistics. Bone Marrow Transplant. 2013:48,452-458. advance online publication 3 December 2012; doi: 10.1038/bmt.2012.244

[12] Schwartz DL, Rajendran J, Yueh B, et al. FDG-PET prediction of head and neck squamous cell cancer outcomes. Arch Otolaryngol Head Neck Surg 2004;130:1361-67.

[13] Machtay M, Natwa M, Andrel J, Hyslop T, Anne PR, Lavarino J, et al. Pretreatment FDG-PET standardized uptake value as a prognostic factor for outcome in head and neck cancer. Head Neck. 2009;31(2):195-201.

[14] Torizuka T, Tanizaki Y, Kanno T, Futatsubashi M, Naitou K, Ueda Y, et al. Prognostic value of 18F-FDG PET in patients with head and neck squamous cell cancer. AJR Am J Roentgenol. 2009;192(4):W156-60.

[15] Kinahan PE, Doot RK, Wanner-Roybal M, Bidaut LM, Armato SG, Meyer CR, et al. PET/CT assessment of response to therapy: tumor change measurement, truth data, and error. Transl Oncol. 2009;2(4):22330.

[16] Fahey FH, Kinahan PE, Doot RK, Kocak M, Thurston H, Poussaint TY. Variability in PET quantitation within a multicenter consortium. Med Phys. 2010;37(7):3660-6.

[17] Brasse D, Kinahan PE, Lartizien C, Comtat C, Casey M, Michel C. Correction methods for random coincidences in fully 3D whole-body PET: impact on data and image quality. J Nucl Med. 2005;46(5):859-67.

[18] Chung MK, Jeong HS, Park SG, et al. Metabolic tumor volume of [18F]-fluorodeoxyglucose positron emission tomography/computed tomography predicts short-term outcome to radiotherapy with or without chemotherapy in pharyngeal cancer. Clin. Cancer Res. 2009; 15(18):5861-5868.

[19] Hatt M, Visvikis D, Pradier O, Cheze-Le Rest C. Baseline (1)(8)F-FDG PET image-derived parameters for therapy response prediction in oesophageal cancer. Eur. J. Nucl. Med. Mol. Imag. 2011; 38(9):15951606

[20] Romesser PB, Qureshi MM, Shah BA, et al. Superior prognostic utility of gross and metabolic tumor volume compared to standardized uptake value using PET/CT in head and neck squamous cell carcinoma patients treated with intensity-modulated radiotherapy. Ann. Nucl. Med. 2012; 26(7):527-534.

[21] La TH, Filion EJ, Turnbull BB. Metabolic Tumor Volume Predicts for Recurrence and Death in Headand-Neck Cancer. Int J Radiat Oncol Biol Phys. 2009. 


\section{Tables}

Table 1. Patients` characteristics

\begin{tabular}{lll}
\hline Age (years) & $40-91$ & (median 61) \\
Gender & & $(85.7 \%)$ \\
Male & 54 & $(14.3 \%)$ \\
Female & 9 & \\
Histology & & $(96.8 \%)$ \\
Squamous cell carcinoma & 61 & $(3.2 \%)$ \\
Undifferentiated carcinoma & 2 & \\
Tumor location & & \\
Oropharynx & 23 & $(36.5 \%)$ \\
Hypopharynx & 39 & $(61.9 \%)$ \\
Larynx & 1 & $(1.6 \%)$ \\
Stage (6th ed.) & 5 & $(7.9 \%)$ \\
I & 7 & $(11.1 \%)$ \\
II & 8 & $(12.7 \%)$ \\
III & 43 & $(68.3 \%)$ \\
IV & & \\
Total dose of radiotherapy & 70 & \\
(Gy) & \\
Chemotherapy regimen. & & \\
CDDP or CBDCA & 51 & $(81.0 \%)$ \\
Other & 2 & $(3.2 \%)$ \\
RT alone & 10 & $(15.9 \%)$ \\
Neoadjuvant chemotherapy & 14 & $(22.2 \%)$ \\
FP & 14 & $(22.2 \%)$ \\
TPF & 7 & $(11.1 \%)$ \\
Neck Dissection & & \\
\hline
\end{tabular}

Abbreviations: $\mathrm{CDDP}=$ cisplatin, $\mathrm{CBDCA}=$ carboplatin, $\mathrm{FP}=$ cisplatin $+5-\mathrm{FU}, \mathrm{TPF}=$ docetaxel + cisplatin $+5-\mathrm{FU}$

Table 2 PET/CT parameters, and survival and disease control status

Locoregional control Distant control Overall survival Disease-free survival

\begin{tabular}{|c|c|c|c|c|c|}
\hline Median & $\mathbf{n}$ & \multicolumn{4}{|c|}{ Events (5-year actuarial control rates) } \\
\hline All & 63 & $72.7 \%$ & $82.0 \%$ & $58.2 \%$ & $63.0 \%$ \\
\hline $\begin{array}{l}\text { SUVmax } \\
<14.8 \\
>14.8 \\
\text { p value }\end{array}$ & $\begin{array}{l}45 \\
18\end{array}$ & $\begin{array}{l}74.3 \% \\
68.4 \% \\
0.643\end{array}$ & $\begin{array}{l}85.3 \% \\
73.1 \% \\
0.381\end{array}$ & $\begin{array}{l}64.4 \% \\
41.9 \% \\
0.071\end{array}$ & $\begin{array}{l}68.5 \% \\
50.0 \% \\
0.171\end{array}$ \\
\hline $\begin{array}{l}\text { TBR } \\
<8.7 \\
>8.7 \\
\text { p value } \\
\text { MTV }\end{array}$ & $\begin{array}{l}44 \\
19\end{array}$ & $\begin{array}{l}75.8 \% \\
65.6 \% \\
0.233\end{array}$ & $\begin{array}{l}86.6 \% \\
71.5 \% \\
0.164\end{array}$ & $\begin{array}{l}61.3 \% \\
50.5 \% \\
0.397\end{array}$ & $\begin{array}{l}67.7 \% \\
52.6 \% \\
0.158\end{array}$ \\
\hline $\begin{array}{l}<5.4 \mathrm{ml} \\
>5.4 \mathrm{ml} \\
\text { p value }\end{array}$ & $\begin{array}{l}49 \\
14\end{array}$ & $\begin{array}{l}78.7 \% \\
43.2 \% \\
0.025\end{array}$ & $\begin{array}{l}88.7 \% \\
60.6 \% \\
0.008\end{array}$ & $\begin{array}{l}66.7 \% \\
28.6 \% \\
0.002\end{array}$ & $\begin{array}{l}73.1 \% \\
28.6 \% \\
<0.001\end{array}$ \\
\hline $\begin{array}{l}\text { < } 38.5 \mathrm{ml} \\
>38.5 \mathrm{ml} \\
\text { p value }\end{array}$ & $\begin{array}{l}43 \\
20\end{array}$ & $\begin{array}{l}80.4 \% \\
55.5 \% \\
0.029\end{array}$ & $\begin{array}{l}87.5 \% \\
67.6 \% \\
0.104\end{array}$ & $\begin{array}{l}67.4 \% \\
37.7 \% \\
0.013\end{array}$ & $\begin{array}{l}74.0 \% \\
40.0 \% \\
0.005\end{array}$ \\
\hline
\end{tabular}

\section{Figures}



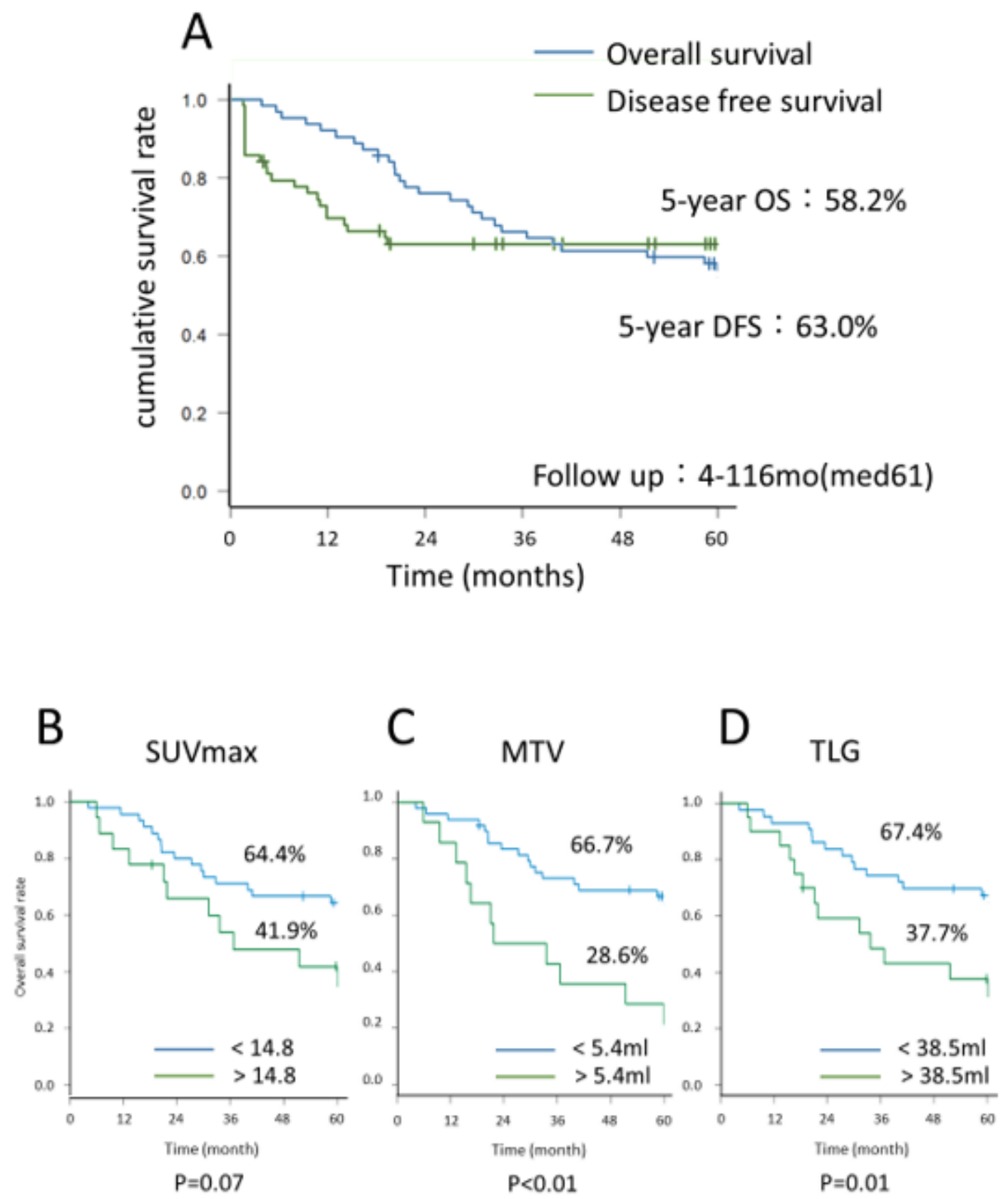

Figure 1

Overall survival according to PET/CT parameters.

A-D, Kaplan Meier survival curves with all patients (A), and between groups for maximum standardized uptake value (SUVmax) (B), metabolic tumor volume (MTV), and total lesion glycolysis (TLG) (D).

\section{Figure 2}




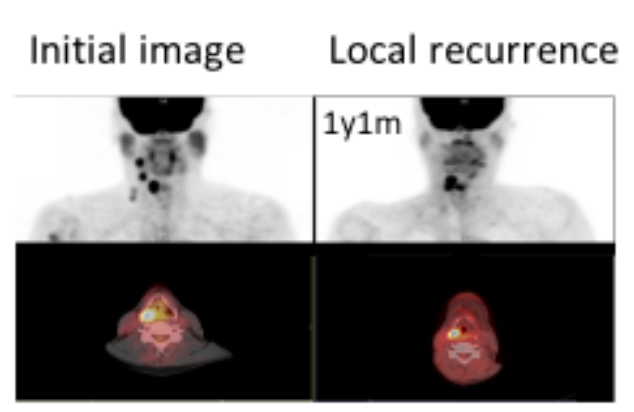

HPC T2N2bMO

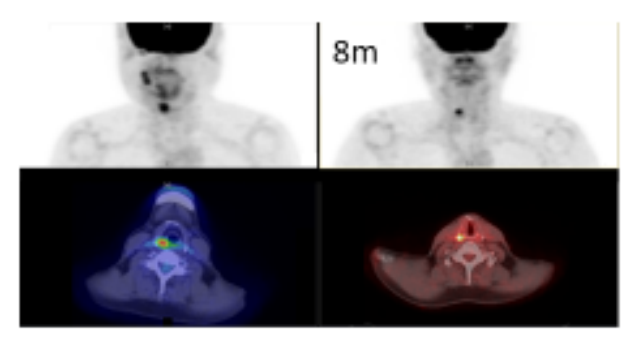

HPC T4aN2cM0

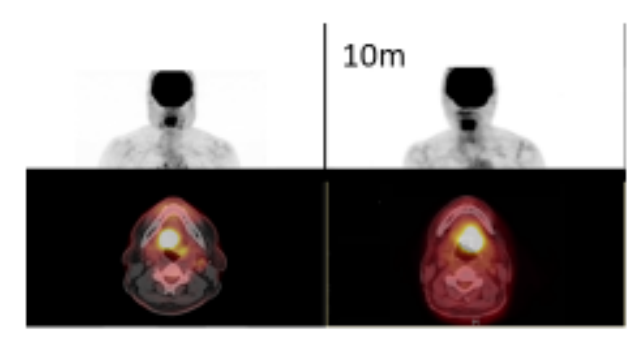

OPC T4aNOMO

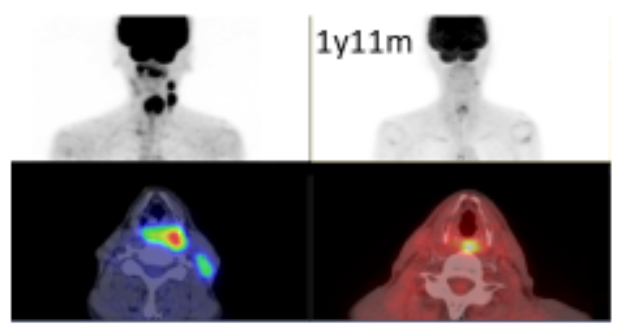

HPC T1N2bM0

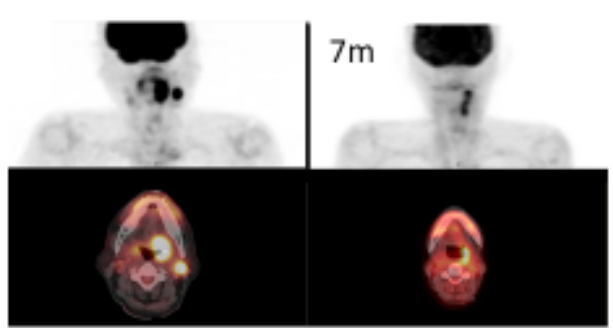

HPC T4aN1M0

Figure 3

Representative images of patients with local recurrence after definitive IMRT. 